\title{
POSSIBLE USE OF SMART ANTENNAS FOR DEVELOPMENT OF INTELLIGENT COMMUNICATION SYSTEMS
}

\author{
Sridhar Pattanaik. ${ }^{1}$, R.K Mishra ${ }^{2}$ \\ ${ }^{1,2}$ Department of Electronic Science, Berhampur University, Berhampur, India \\ Email: 'sridhar_pattanaik@yahoo.co.in
}

\begin{abstract}
Intelligent systems are nature-inspired, mathematically sound, computationally intensive problem solving tools and methodologies that have become important for future communication. Intelligent systems will enable safe, cost and missioneffective approaches to meet the technical specifications. SS7, INAP, CAMEL, WIN, GSM MAP, and ANSI-41 were used in traditional intelligence in mobile. Mobile networks are evolving beyond traditional network intelligence and known as Intelligent Mobile Network. Due to the advancement of technologies such as smart antennas and intelligent BSS/OSS systems are deployed to provide greater efficiencies and more effective network operations and as a result the network is becoming more intelligent. Next generation networks of the near future will leverage all of these technologies in conjunction with traditional network intelligence for more efficient and effective operations as well as the provision of advanced and value-added services. In this paper the use of smart in the possible development of intelligent communication systems is discussed.
\end{abstract}

Key words: SmartAntenna, DSP processor, 3G, SS7, INAP, CAMEL, WIN, GSM MAP

\section{INTRODUCTION}

The broadband wireless communication systems require the development of antennas that are small and affordable to a large number of users. These antennas must also go broadband to provide newer generation technology the possibility of offering more capacity to the busiest cell sites or create specific beams for each mobile user while continuously adapting to the changing environment (1-2).

Possible smart antennas (1) offers less interference from other users and effectively boost the current generation network in the $3-G$ systems. In other applications, such as the future indoor systems, millimetre waves is able to offer a large bandwidth with the additional benefit of mobility and cellular strategies enabling substantial amounts of information to be distributed simultaneously to many users of a significant area. These systems will provide to the subscribers with a vast array of voice, data, image and multimedia services. The development of appropriate smart array and associated technology will be important to the success of broadband wireless systems deployment at millimeter wave frequencies. Working at these frequencies presents new challenges, both at the propagation and at the circuitry and antennas levels.

\section{ANTENNA TECHNOLOGY}

Antenna determines the manner in which energy is distributed into and collected from surrounding space. The service quality provided by the network is determined by the antenna system. It has a greater influence on the efficient use of spectrum, the cost of establishing new networks.
The simple dipole antenna radiates and receives equally well in all directions and was used in the early days for wireless communications. These are adequate for simple RF environment where no specific knowledge of the users' whereabouts is known. Only a small percentage of the overall energy sent out into the environment is being used in the unfocused approach. The omni-directional strategies attempt to overcome environmental challenges by simply boosting the power level of the signals to be broadcast.

This unfocused approach results in only a small percentage of the overall energy sent out into the environment. In case of numerous users the interference in the same or adjoining cells becomes prominent. Spectral efficiency, limiting frequency reuse is also affected due to the omni-directional strategies. These lacunas force system designers and network planners to devise increasingly sophisticated and costly remedies.

In recent years, the limitations of broadcast antenna technology on the quality, capacity, and coverage of wireless systems have prompted an evolution in the fundamental design and role of the antenna in a wireless system (3-4).

A single antenna can also be constructed to have certain fixed preferential transmission and reception directions. Many conventional antenna towers today split, or sectorize cells. A $360^{\circ}$ area is often split into three $120^{\circ}$ subdivisions, each of which is covered by a slightly less broadcast method of transmission.

An antenna can be made intelligent by modifying the physical design by adding more elements. An antenna system is designed to shift signals before transmission at 
each of the successive elements, which has a composite effect, and this hardware and software concept is known as the phased array antenna. Sectorized and diversity systems are the two basic types of antenna systems. The former take a traditional cellular area and subdivide it into sectors that are covered using directional antennas and the latter incorporates two antenna elements at the base station using space diversity. Diversity offers an improvement in the effective strength of the received signal by using any one of the methods namely switched diversity or diversity combining.

Diversity antennas are switch operated from one working element to another this antenna mitigates severe multipath fading. The use of one element at a time offers no uplink gain improvement over any other single element approach. In high-interference environments, locking onto the strongest signal is clearly inappropriate and can result in crystal-clear reception of an interferer rather than the desired signal. The need to transmit to numerous users more efficiently without compounding the interference problem led to the next step of the evolution antenna systems that intelligently integrate the simultaneous operation of diversity antenna elements.

\section{III.FUNDAMENTALS OF SMART ANTENNA}

The concept of using multiple antennas and innovative signal processing to serve cells more intelligently has existed for many years. The advent of powerful low-cost digital signal processors (DSPs), general-purpose processors (and ASICs), as well as innovative software-based signal-processing techniques (algorithms) have made intelligent antennas practical for cellular communications systems. Today, when spectrally efficient solutions are increasingly a business imperative, these systems are providing greater coverage area for each cell site, higher rejection of interference, and substantial capacity improvements.

Generally collocated with a base station, a smart antenna system (1) combines an antenna array with a digital signal-processing capability to transmit and receive in an adaptive, spatially sensitive manner. In other words, such a system can automatically change the directionality of its radiation patterns in response to its signal environment. This can dramatically increase the performance characteristics of a wireless system and can make the network intelligent.

\section{SMART ANTENNA SYSTEMS}

Smart antenna systems are customarily categorized as switched beam and adaptive array systems. The two major categories of smart antennas regarding the choices in transmit strategy are switched beam and adaptive array are discussed next.
In switched beam a finite number of fixed, predefined patterns or combining sectors exists. These antenna systems are direction sensitive with multiple fixed beams. It detects signal strength from one of several predetermined, fixed beams, and switch from one beam to another as the mobile moves throughout the sector. Switched beam systems combine the outputs of multiple antennas in such a way as to form finely directional beams with more spatial selectivity than can be achieved with conventional, single-element approaches.

In Adaptive Array antennas new signal-processing algorithms are utilized to effectively locate and track various types of signals to dynamically minimize interference and maximize intended signal reception. Both systems i.e. switched and adaptive attempts to increase gain according to the location of the user; however, only the adaptive system provides optimal gain while simultaneously identifying, tracking, and minimizing interfering signals. Adaptive antenna technology represents the most advanced smart antenna approach to date.

Omni-directional antennas are obviously distinguished from their intelligent counterparts by the number of antennas (or antenna elements) employed. Switched beam and adaptive array systems, however, share many hardware characteristics and are distinguished primarily by their adaptive intelligence. To process information that is directionally sensitive requires an array of antenna elements (typically 4 to 12), the inputs from which are combined to control signal transmission adaptively. Antenna elements can be arranged in linear, circular, or planar configurations and are most often installed at the base station, although they may also be used in mobile phones or laptop computers.

An antenna is expected to work in a RF environment. When the environment is complex the number of users, interference, and propagation complexity grow and smart antenna solutions are required. The digital signalprocessing facilities make them smart. Accuracy and flexibility of operation are the features of manipulating the RF data in digital format. For Smart antenna transmitting systems the steps involved are capture, convert, and modulate analog signals as digital signals and reconvert them to analog information on the other end.

\section{DESTINATIONS OF SMART ANTENNA SYSTEMS}

Augment the signal quality of the radio-based system through more focused transmission of radio signals while enhancing capacity through increased frequency reuse are the purposes of smart antenna systems (3-5). 
Signal gain leads to better coverage is achieved by multiple antennas combined to optimize available power to establish given level of coverage. Utilizing the focusing techniques increases the base station range and coverage. As a result the power requirements is reduced and enable a greater battery life and smaller/lighter handset size. Antenna pattern can be generated toward co-channel interference sources, improving the signal to-interference ratio of the received signals. Spatial diversity can reduce the effective delay spread of the channel, allowing higher bit rates to be supported without the use of an equalizer. Power efficiency will lead to reduce expenses.

\section{ARCHITECTURE OF SMART ANTENNA SYSTEMS}

In switched beam and adaptive array systems enable a base station to customizes the beams they generate for each remote user effectively by means of internal feedback control this approach is traditional. The main objective of any approach is to form a main lobe toward individual users and attempts to reject interference or noise from outside of the main lobe. The uplink and down link technique for smart antenna system is discussed next.

In the uplink process it is assumed here that a smart antenna is only employed at the base station and not at the handset or subscriber unit. Such remote radio terminals transmit using omnidirectional antennas, leaving it to the base station to separate the desired signals from interference selectively. The received signal from the spatially distributed antenna elements is multiplied by a weight, a complex adjustment of an amplitude and a phase. These signals are combined to yield the array output. Adaptive algorithm controls the weights according to predefined objectives. In case of switched beam system, this may be primarily maximum gain; for an adaptive array system (5-7), other factors may receive equal consideration. These dynamic calculations enable the system to change its radiation pattern for optimized signal reception.

In the downlinking process the task of transmitting in a spatially selective manner. The major basis for differentiating between switched beam and adaptive array systems. Switched beam systems communicate with users by changing between preset directional patterns, largely on the basis of signal strength. Where as adaptive arrays attempt to understand the RF environment more comprehensively and transmit more selectively. The type of downlink processing used depends on duplex. If the communication system uses time division duplex (TDD), in which transmission and reception is done at the same frequency otherwise, frequency division duplex (FDD), is utilized which uses separate frequencies for transmitting and receiving. In most FDD systems the propagation characteristics is be considered independent. In case of TDD systems the uplink and downlink channels can be considered reciprocal. In TDD systems the uplink channel information may be used to achieve spatially selective transmission where as it cannot be used directly in its FDD counterpart. In case of FDD systems, other types of downlink processing must be considered.

Switched beam is an extension of the current microcellular or cellular sectorization method of splitting a typical cell, if we speak in terms of radiation pattern. In order to improve the range and capacity the switched beam approach further subdivides macro-sectors into several micro-sectors. Each micro-sector contains a predetermined fixed beam pattern. The design of such systems involves high-gain, narrow azimuthal beamwidth antenna elements. The switched beam system selects one of several predetermined fixed-beam patterns and the choices are driven by RF or baseband DSP hardware and software. The system switches its beam in different directions throughout space by changing the phase differences of the signals used to feed the antenna elements or received from them. The switched beam system selects the micro-sector containing the strongest signal when the mobile user enters a particular macrosector. Throughout the call, the system monitors signal strength and switches to other fixed micro-sectors as required. Smart antenna systems communicate directionally by forming specific antenna beam patterns. When a smart antenna directs its main lobe with enhanced gain in the direction of the user, it naturally forms side lobes and nulls in directions away from the main lobe. Controlling the lobes and the nulls with varying degrees of accuracy and flexibility is done by different switched beam and adaptive smart antenna systems.

In Adaptive Antenna Approach communication between a user and base station in done in a different way. Adaptive antenna technology can dynamically alter the signal patterns to optimize the performance of the wireless system when the RF environment is changed. Sophisticated signal-processing algorithms is used continuously to distinguish between desired signals, multipath, and interfering signals as well as calculate their directions of arrival. Based on changes in both the desired and interfering signal locations it continuously updates it's transmit strategy. The ability to track users smoothly with main lobes and interferers with nulls ensures that the link budget is constantly maximized. The interference rejection capability of the adaptive system provides significantly more coverage than either the conventional or switched beam system.

The comparison of the two systems can be broadly done on the basis of integration, range, interference 
suppression etc. In comparison, adaptive array systems have been deployed with a more fully integrated approach that offers less hardware redundancy than switched beam systems but requires new build-out. Depending on environmental circumstances and the hardware/software used Switched beam systems can increase base station range from 20 to 200 percent over conventional sectored cells. The added coverage can save an operator substantial infrastructure costs and means lower prices for consumers. The dynamic switching conserves capacity as the system need not send all signals in all directions. Adaptive array systems can cover a broader, more uniform area with the same power levels as a switched beam system.

Interference arriving from directions away from the active beam's center are suppressed by the switched beam antennas. Switched beam solutions work best in minimal to moderate co- channel interference and have difficulty in distinguishing between a desired signal and an interferer.

Adaptive array technology currently offers more comprehensive interference rejection as it transmits an infinite number of combinations and its narrower focus creates less interference to neighboring users than a switched-beam approach.

The most sophisticated utilization of smart antenna technology (1-4) is spatial division multiply access (SDMA), which employs advanced processing techniques to, in effect, locate and track fixed or mobile terminals, adaptively steering transmission signals toward users and away from interferers. This scheme can adapt the frequency allocations to where the most users are located. Spatial processing dynamically creates a different sector for each user and conducts a frequency/channel allocation in an ongoing manner in real time. A higher level of measurement and analysis of the scattering aspects of the RF environment is integrated in the adaptive spatial processing. In case of traditional beam forming and beamsteering techniques assume one correct direction of transmission toward a user; spatial processing maximizes the use of multiple antennas to combine signals in space in a method that transcends a one user-one beam methodology.

\section{SIGNAL PROCESSING TECHNIQUE}

Appropriate signal-processing techniques to be implemented using a reasonable amount of digital electronics. The work of the signal may be Spatial filtering with smart antennas. When dealing with beam-forming type, it could be fixed beams or adaptive beams with SNR or Maximum likelihood or Minimum Mean squared error while for adaptive optimal combining it involves maximum
SNR, maximum MSE (mean square error), minimum variance distorsionless response and many others.

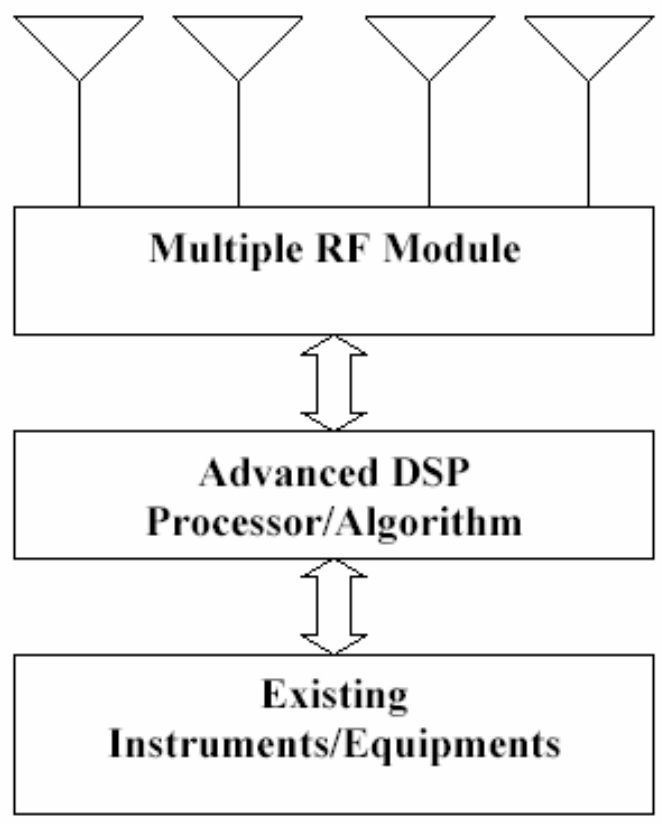

Fig. 1. Intelligent antennas as spatial filter

Physical implementation of the intelligence with the antenna is a challenge, particularly at high frequencies. Intelligent antenna system has the potential to separate spectrally and temporally overlapping signals, which enables them for use in the CDMA, TDMA or FDMA systems. It can enhance or reject signals based on their direction of arrival. Intelligent antenna system combines the outputs of multiple antenna elements with signal processing capabilities to transmit and/or receive RF signals. The $T x / R x$ is done in an adaptive, spatially sensitive manner. It requires a frequency translator from RF to base band for signal processing.

\section{POTENTIAL APPLICATION OF SMART ANTENNA}

Smart antenna technology can significantly improve wireless system performance and economics for a range of potential users. It enables operators of PCS, cellular, and wireless local loop (WLL) networks to realize significant increases in signal quality, capacity, and coverage. Smart antenna systems are applicable, with some modifications, to all major wireless protocols and standards. Frequency division multiple access (FDMA) (e.g., AMPS, TACS, NMT) can be used for the analog access where as time division multiple access (TDMA) (e.g., GSM, IS-136); code division multiple access (CDMA) (e.g., IS-95) are the digital access technique which can be utilized. FDD (frequency division duplex) and TDD (time division duplex) can be used for the intelligent antenna systems. 


\section{CONCLUSION}

The flexibility of adaptive smart antenna technology allows for the creation of new value-added products and services that give operators a significant competitive advantage. Adaptive smart antennas are not restricted to any particular modulation format or air-interface protocol. They are compatible with all current air-interface modulation schemes.

A wide range of wireless communication systems may benefit from spatial processing, including high-mobility cellular systems, low-mobility short-range systems, wireless local loop applications, satellite communications, and wireless LAN. By employing an array of antennas, it is possible to multiplex channels in the spatial dimension just as in the frequency and time dimensions. To increase system capacity, spatially selective transmission as well as spatially selective reception must be achieved. Improved algorithms and low-cost processors make sophisticated spatial processing practical alternatives for an increasing number of wireless systems.

\section{REFERENCES}

[1] G.V. Tsoulos, April 1999,"Smart antennas for mobile communication systems: benefits and challenges", Electronics and communications Engineering International Journal, vol. II, no. 2, pp. 84-94.

[2] Y. Qian and T. Itoh, Nov. 1998, "Progress in active integrated antennas and their applications," IEEE Trans. Microwave Theory Tech., vol. 46, pp. 18911900.

[3] J.-Y. Park, Y. Wang, and T. Itoh, 2003," A 60 GHz integrated antenna array for high-speed digital beamforming applications," IEEE International Microwave Symposium Digest, vol. 3, pp. 1677-1680.
[4] M. Devincentis and T. Itoh, 2003, "A $40 \mathrm{GHz}$ communication link with IF-assisted self-heterodyne direct down conversion," IEEE International Microwave Symposium Digest, vol. 1, pp. 91-94.

[5] R. Y. Miyamoto, Y. Qian, and T. Itoh, 2001 ,A reconfigurable active retrodirective/direct conversion receiver array for wireless sensor systems," IEEE MTT-S Intl. Microwave Symposium Digest, pp. 11191122.

[6] M. Iskander, Z. Yun, Z. Zhang, R. Isam, Sept. 10-14, 2001, "Antenna technology and propagation models for the next generation wireless communication systems", International Conference on Electromagnetics in Advanced Applications, (ICEAA'01), Torino, Italy, pp. 787-790.

[7] K. Hettak, G.Y. Delisle, July 16-21, 2000,"A novel variant of dual polarized CPW fed patch antenna for broadband wireless communications", IEEE AP-S International Symposium, Salt Lake City, Utah, pp. 286-289.

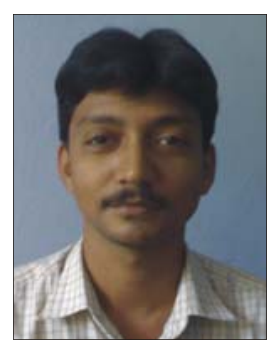

Sridhar Pattanaik is teaching in the Department of Electronic Science Berhampur University. He has published more than 20 research articles; his research interest includes Microstrip Antenna, ANN, CEM, Smart antennas. 